\title{
Electromagnetic Launch to Space
}

\author{
D. Wetz*, I. McNab, F. Stefani and J. Parker \\ Institute for Advanced Technology, The University of Texas at Austin \\ 3925 W. Braker Lane, Ste. 400, Austin, TX 78759, USA
}

\begin{abstract}
Many advances in electromagnetic (EM) propulsion technology have occurred in recent years. Linear motor technology for low-velocity and high-mass applications is being developed for naval catapults and missile launchers. Such technology could serve as the basis for the launch of a first-stage booster launch - for example, as suggested some years ago by the US National Aeronautics and Space Administration (NASA) in the Maglifter concept. For higher velocities, experimental laboratory railguns have demonstrated launch velocities of $2-3 \mathrm{~km} / \mathrm{s}$ and muzzle energies greater than $10 \mathrm{MJ}$. The extension of this technology to the muzzle velocities $(\geq 7500 \mathrm{~m} / \mathrm{s})$ and energies (hundreds of megajoules) needed for the direct launch of payloads into orbit is very challenging but may not be impossible. For launch to orbit, long launchers $(>1000 \mathrm{~m})$ would need to operate at accelerations $>1000 \mathrm{G}$ to reach the required velocities, so it would only be possible to launch rugged payloads, such as fuel, water, and material. This paper provides an overview of these concepts and includes a summary of the recent advances made in this area.
\end{abstract}

PACS numbers: 96.12.Hg, 98.35.Eg, 94.30.Kq, 94.05.Rx, 94.20.Fg, 94.20.wc, 94.20.wf

\section{Introduction}

Due to the constantly increasing cost of using conventional rocket technology to place a microsatellite (1-10 kg) into low-Earth orbit (LEO), both government and industry are interested in alternative methods to launch to space. The use of EM launch is one possible alternative. Over the last several decades, a great deal of research has been conducted on EM launchers for use in both tactical (using conventional solid armatures) and launch-to-space applications (using a plasma armature). Solid-armature railguns are typically used to launch payloads to muzzle velocities no higher than $3 \mathrm{~km} / \mathrm{s}$ with muzzle energies in the tens of megajoules. These types of guns are typically considered for tactical applications. Velocities in excess of $7 \mathrm{~km} / \mathrm{s}$ are required for launch to space; therefore, solid-armature guns are not applicable. For this type of application, plasma armatures are required.

A significant amount of research was performed in the 1970s and early 1980s to investigate the design of plasma-driven EM launchers. Researchers involved in that research were met with a great deal of challenge and difficulty, especially in getting plasma armatures to accelerate payloads to muzzle velocities in excess of $6 \mathrm{~km} / \mathrm{s}$ at acceleration levels under $1 \mathrm{MG}$ [1]. In those experiments, velocities of only $4-5 \mathrm{~km} / \mathrm{s}$ were achieved for medium-bore $(25-50 \mathrm{~mm})$ railguns operating at typical accelerations of $400-600 \mathrm{kG}[2,3]$, and velocities of $6-7 \mathrm{~km} / \mathrm{s}$ were achieved in smaller bore guns operating at $1 \mathrm{MG}$ or greater $[4,5]$. The researchers involved proposed a

\footnotetext{
* corresponding author; e-mail: david_wetz@iat.utexas.edu
}

number of theories to account for the velocity ceiling and even proposed a number of solutions that could be used to overcome the obstacles, but no significant effort was put into implementing them. The reason for the velocity ceiling was found to be a direct consequence of ablation of the bore insulators, which causes the bore to fill up with a hot, dense, neutral gas [6]. The gas does not affect the performance of the railgun until, at high velocities, the voltage across the railgun breech increases to the point where conditions for high-voltage breakdown are met. When this occurs, additional plasma armatures, called restrike or secondary arcs, are formed well behind the main armature. These secondary armatures are retarded by viscous drag as they push the ablation products created in the launcher bore. This drag prevents the restrike arcs from catching up to the main armature, causing current and acceleration force to be lost in the restrike arc, thereby preventing further acceleration of the payload.

Research in this area has recently been revived by researchers at the Institute for Advanced Technology (IAT) in Austin, Texas, USA, as part of multidisciplinary university research initiative (MURI) supported by the US Air Force Office of Scientific Research (AFOSR). The IAT's program focus is directed toward developing a subscale, proof-of-principle EM launcher capable of accelerating $5-10 \mathrm{~g}$ polycarbonate projectiles up to muzzle velocities in excess of $7 \mathrm{~km} / \mathrm{s}$. In order to overcome the velocity ceiling, the experimental setup at the IAT is designed to practically eliminate bore wall ablation. This is achieved using three main techniques:

1. magnetic augmentation to reduce power dissipation in the plasma, 
2. high-purity alumina insulators to raise the ablation resistance of the bore, and

3. pre-acceleration to prevent ablation of the bore materials at low velocity.

A thorough documentation of the experimental design and the initial round of testing are best documented in [7]. This paper focuses on recent improvements based on conclusions drawn from the initial round of testing.

\section{Experimental setup}

The initial round of full-scale testing at the IAT was performed in mid 2007. In those tests, peak velocities of 4.2 and $5.2 \mathrm{~km} / \mathrm{s}$ were achieved in 3.1 and $7 \mathrm{~m}$ long guns, respectively. Those tests were considered very successful, in that such high velocities were achieved with no evidence of restrike phenomena, but unsuccessful in the sense that most of the ceramic insulators were broken during each of the shots taken. Though such breakage is irrelevant to achieving $7 \mathrm{~km} / \mathrm{s}$, buying a full build of ceramic insulators for each test is far too expensive to conduct a significant amount of research. Because the plasma armature exerts a uniform pressure of $100 \mathrm{MPa}$ on the inner bore walls as it travels down the bore, it is imperative that the tiles be put into compression at an equal pressure in both the horizontal and vertical directions prior to shooting. The original launcher design did not provide sufficient precompression to prevent the tiles from breaking. To correct this problem, several changes have been made to the railgun core, shown in Fig. 1.

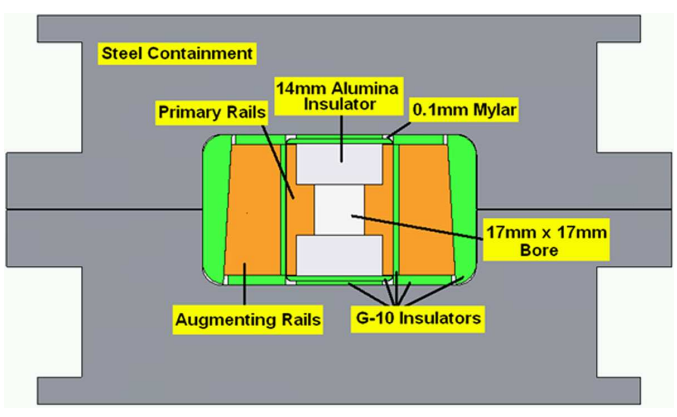

Fig. 1. Modified version of the IAT plasma railgun core.

The changes from the original core design can be summarized as follows: an increased tile thickness from $\sim 7 \mathrm{~mm}$ to $\sim 14 \mathrm{~mm}$, a taller inner rail to accommodate the thicker tile, a tapered augmenting rail, and a $0.1 \mathrm{~mm}$ thick Mylar overwrap instead of polyolefin heat shrink. The increased tile thickness increases the tile stiffness by a factor of seven. In addition to the tiles being thicker, their overall length has been reduced to $5 \mathrm{~cm}$. The addition of a taper to the augmenting rails and G-10 side insulators provides an increased stroke so that the necessary precompression can be applied without sheering the side insulators. The required amount of interference needed to obtain adequate precompression has been significantly reduced by changing the overwrap to Mylar, which is thinner and stiffer, instead of heat shrink, which is almost six times thicker and much spongier.

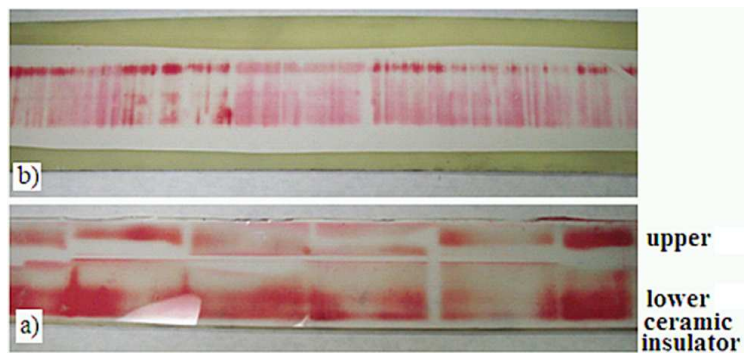

Fig. 2. High Pressurex ${ }^{\circledR}$ film between the (a) upper G-10 insulator and steel containment and (b) primary and augmenting rails.

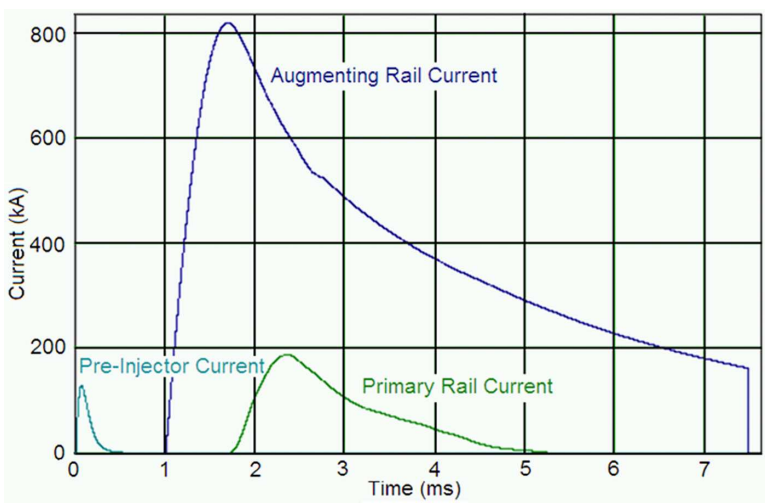

Fig. 3. Shot currents.

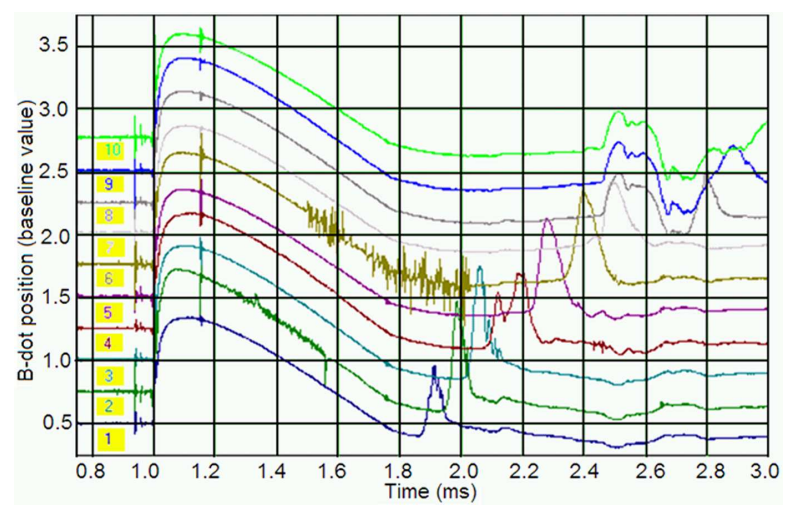

Fig. 4. B-dot traces in a waterfall plot.

In order to verify the tiles are being adequately compressed, Pressurex film has been integrated into the experiment. The film has ink modules between the layers of film that pop when pressure is applied, leaving a colored pattern proportional to the amount of pressure applied. Figure 2 displays a sample of High Pressurex film, which 
measures between 49 and $128 \mathrm{MPa}$, located between the augmenting and inner rails as well as between the G-10 top insulator and the top containment. The figures show that an acceptable uniform compression, $\sim 100 \mathrm{MPa}$, is being applied in all directions. All other aspects of the experiment have remained unchanged from the initial design.

\section{Experimental results}

The first round of testing on the modified core design began in the spring of 2008. Figure 3 displays the currents measured during the most recent shot taken on a $3.1 \mathrm{~m}$ long gun. Figure 4 plots the B-dot traces in a waterfall plot, wherein the baseline voltage of each trace corresponds to its position along the gun. Figure 3 shows that just over $800 \mathrm{kA}$ is run through the augmenting rails and just under $200 \mathrm{kA}$ is flowing through the plasma armature. It should be noticed in Fig. 4 that there is no evidence of restrike arc phenomena occurring. The traces recorded on B-dots 8-10 are not entirely understood as they all appear to turn on at the same time, which is not normal behaviour. It is believed that as the armature approached the end of the gun, the muzzle cap blew up, disrupting the vacuum conditions at the end of the gun and making it possible for a second arc to form at the muzzle. It is possible that the arc acts as a low-impedance muzzle shunt that steals current from the armature, causing a decrease in the velocity. Despite that, a peak average velocity of roughly $2.75 \mathrm{~km} / \mathrm{s}$ is achieved between B-dots 6 and 7 , located at $1.67 \mathrm{~m}$ and $1.90 \mathrm{~m}$, respectively. Additionally, only two tiles were broken during the shot, indicating that the modified core design has improved the precompression on the ceramic insulators.

\section{Conclusions}

The testing on the modified core design has only recently begun but has already shown a significant im- provement in the ability to make ceramic insulators survive a hypervelocity shot. The future plans are to begin working with a longer gun so that higher velocities, $\sim 7 \mathrm{~km} / \mathrm{s}$, can be obtained.

\section{Acknowledgments}

This material is based upon work supported by the AFOSR under award number FA9550-05-1-0341. The support of the program manager, Mitat Birkan, is gratefully acknowledged. Any opinions, findings, and conclusions or recommendations expressed in this publication are those of the authors and do not necessarily reflect the views of the AFOSR.

\section{References}

[1] S. Rashleigh, R. Marshall, J. Appl. Phys. 49, 2540 (1978).

[2] J.J. Scanlon, J.H. Battek, L.D. Thornhill, K.A. Jamison, D.M. Littrel, IEEE Trans. Magn. 29, 859 (1993).

[3] K. Jamison, D. Littrell, IEEE Trans. Magn. 31, 168 (1995).

[4] N. Kawashima, A. Yamori, M. Yamagisawa, H. Kubo, M. Kohno, S. Teii, IEEE Trans. Magn. 29, 431 (1993).

[5] E. Drobyshevski, S.I. Rozov, B.G. Zhukov, R.O. Kurakin, V.M. Sokolov, IEEE Trans. Magn. 31, 295 (1995).

[6] J. Parker, IEEE Trans. Magn. 25, 418 (1989).

[7] D.A. Wetz, F. Stefani, J.V. Parker, I.R. McNab, IEEE Trans. Magn. 45, 1 (in press). 